\title{
Portal hypertension as the initial manifestation of POEMS syndrome: a case report
}

\author{
Lina $\mathrm{Wu}^{1}$, Yue $\mathrm{Li}^{1 *} \mathbb{D}$, Fang Yao ${ }^{1}$, Chongmei $\mathrm{Lu}^{1}$, Jian $\mathrm{Li}^{2}$, Weixun Zhou ${ }^{3}$ and Jiaming Qian ${ }^{1}$
}

\begin{abstract}
Background: Portal hypertension has a broad differential diagnosis. POEMS syndrome is an uncommon cause of it. POEMS syndrome is a rare disease involving multiple organs. In differential diagnosis of portal hypertension, POEMS syndrome should be considered especially when other symptoms such as numbness, organomegaly, endocrine alteration and skin changes also present, as it is highlighted by our case.

Case presentation: We report a 46-year-old Chinese male, a teacher, presenting with portal hypertension. Electromyography revealed peripheral neuropathy. Immunofixation showed monoclonal immunoglobulin A lambda protein. The diagnosis of POEMS syndrome was established. After treatment of lenalidomide combined with dexamethasone over 2 years, the patient achieved a considerable improvement.

Conclusion: This case highlights the manifestation of portal hypertension in POEMS syndrome. Lenalidomide with or without dexamethasone is effective for portal hypertension due to POEMS syndrome, though esophageal and gastric varices seems not reversible so easily.
\end{abstract}

Keywords: Portal hypertension, POEMS syndrome, Castleman disease

\section{Background}

POEMS syndrome is a rare kind of paraneoplastic syndrome and belongs to plasma cell disorder. POEMS is the abbreviation of polyradiculoneuropathy, organomegaly, endocrinopathy, monoclonal plasma cell disorder and skin changes, which is first proposed by Bardwick in 1980 [1]. POEMS syndrome initially presenting as portal hypertension is seldom [2,3]. We reported a rare case of portal hypertension appearing as the first manifestation and aggravating during the course of POEMS syndrome. In differential diagnosis of portal hypertension, POEMS syndrome should be considered especially when other symptoms such as numbness, organomegaly, endocrine alteration and skin changes also present. Since portal hypertension may precede other manifestations of POEMS syndrome by a few years, reevaluation of cryptogenic or

\footnotetext{
* Correspondence: yuelee76@gmail.com

${ }^{1}$ Department of Gastroenterology, Peking Union Medical College Hospital, Chinese Academy of Medical Sciences and Peking Union Medical College, Beijing 100730, China

Full list of author information is available at the end of the article
}

rapidly progressive portal hypertension over time is important.

\section{Case presentation}

A 46-year-old Chinese male was referred to our hospital in September 2013 because of watery diarrhea and refractory ascites for 2 years. Since May 2013, he developed edema and numbness of the lower extremities. He had some difficulty in walking on himself. Two months later, he had dyspnea especially when he stayed prostration. His body weight decreased $15 \mathrm{~kg}$ from October 2010 to October 2011. Due to progressive ascites, his body weight remained stable though leanness continued since then. He had no hematemesis or melena ever before.

Physical exam was notable for cachexia, hyperpigmentation, moderate ascites, jugular venous distention, right axillary lymphadenopathy and splenomegaly. Neurologic examination revealed bilateral lower extremity numbness. The overall neuropathy limitation scale of his arms is 1 , and that of his legs is 2 . The remaining systemic examination was normal. 
Complete blood count was within normal range. Liver biochemical tests and electrolytes were normal except mild hypoalbuminemia ( $23 \mathrm{~g} / \mathrm{l})$. Serum creatinine was elevated $(123 \mu \mathrm{mol} / \mathrm{L})$ and $24 \mathrm{~h}$ urine total protein was $1.6 \mathrm{~g}$. The screening for antinuclear antibodies and autoimmune hepatitis antibodies were negative. Search for parasites and eggs in stools and viral hepatitis in serum were negative. Monoclonal immunoglobulin A lambda protein was detected in the serum by protein electrophoresis and immunofixation. The serum vascular endothelial growth factor (VEGF) level was significantly elevated $(333.1 \mathrm{pg} / \mathrm{ml})$. Endocrine tests confirmed hypothyroidism, hypotestosteronaemia and adrenal insufficiency. Biochemical and cytological analysis of ascitic fluid showed no evidence of infection and malignancy. Serum-ascites albumin gradient (SAAG) is $16 \mathrm{~g} / \mathrm{l}$. Ultrasonography showed reduced flow rate of portal vein, phlebectasia of splenic vein, thrombosis of superior mesenteric vein, suggesting portal hypertension. The depth of ascites was $9.3 \mathrm{~cm}$ measured by ultrasonography. Echocardiography revealed pulmonary artery pressure of $60 \mathrm{mmHg}$. Endoscopy revealed severe-grade esophageal and gastric varices and normal colon. Electromyography found peripheral nerve impaired. CT scan documented lymphadenopathy including retroperitoneal lymph nodes fusion, splenomegaly, and dropsy of multiple serous cavity and established collateral circulation of portal system. Neither nodular contour of the liver nor small liver was revealed. Skeletal radiographs and $\mathrm{PET} / \mathrm{CT}$ revealed lytic lesions with a sclerotic rim on right greater trochanter of femur and densely sclerotic lesions on left ilium. Bone marrow aspirate and biopsy showed plasmocytosis (5\%) with apparently normal morphology. Biopsy of his cervical lymph nodes indicated sinus histiocytosis, which was compatible with Castleman disease (Fig. 1). Histopathology of his liver revealed swelling in part of the hepatocyte, spotty necrosis occasionally and a small quantity of lymphocyte infiltration in portal area, which doesn't support cirrhosis (Fig. 2).

Since the patient presented with polyneuropathy, monoclonal immunoglobulin in immunofixation, Castleman disease, sclerotic bone lesions, serum VEGF elevation,splenomegaly,edema, and endocrinopathy, the diagnosis of POEMS syndrome was established.

After diagnosed as POEMS and Castleman disease, the patient was treated with 12 courses of oral lenalidomide (10 $\mathrm{mg} /$ day) on days $1-21$ plus oral dexamethasone (20 mg/day) on days $1-4$, and 6 courses of oral lenalidomide $(10 \mathrm{mg} /$ day) on days $1-21$. To decrease the risk of thrombosis by lenalidomide, aspirin at $100 \mathrm{mg} /$ day was administered. Thyroxine and prednisone were used for replacement therapy of endocrine disorder. The patient has been on probiotics and diuretics to control watery diarrhea and ascites. He also received physical therapy and exercise as supportive care.

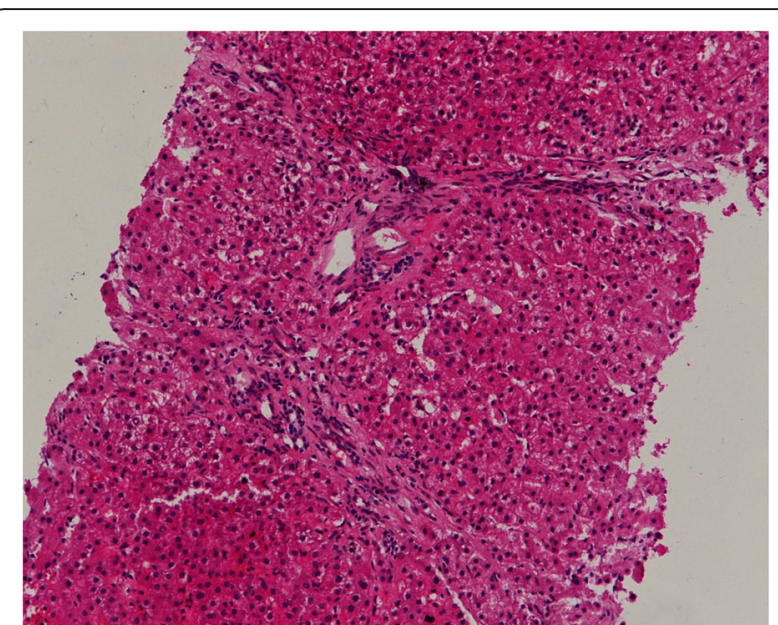

Fig. 1 Biopsy of cervical lymph nodes indicated sinus histiocytosis (Hematoxylin-Eosin staining, 200x). The histopathology was compatible with Castleman disease

There was significant improvement after treatment. Pigmentation of his skin lightened. Abdominal distension and splenomegaly relieved. The depth of ascites decreased to $4.5 \mathrm{~cm}$ after 12 courses of treatment. Numbness of bilateral lower extremities was in remission. The overall neuropathy limitation scale of his arms is 0 , and that of his legs is 0 . M-protein was undetectable by serum protein electrophoresis and immunofixation. Echocardiography revealed that pulmonary artery pressure was normal. Esophageal and gastric varices remained no changes and twice nonfatal hematemesis and melena occurred after eight months treated. Endoscopic variceal ligation therapy was scheduled to prevent variceal bleeding. The patient made a good recovery and went to work as usual.

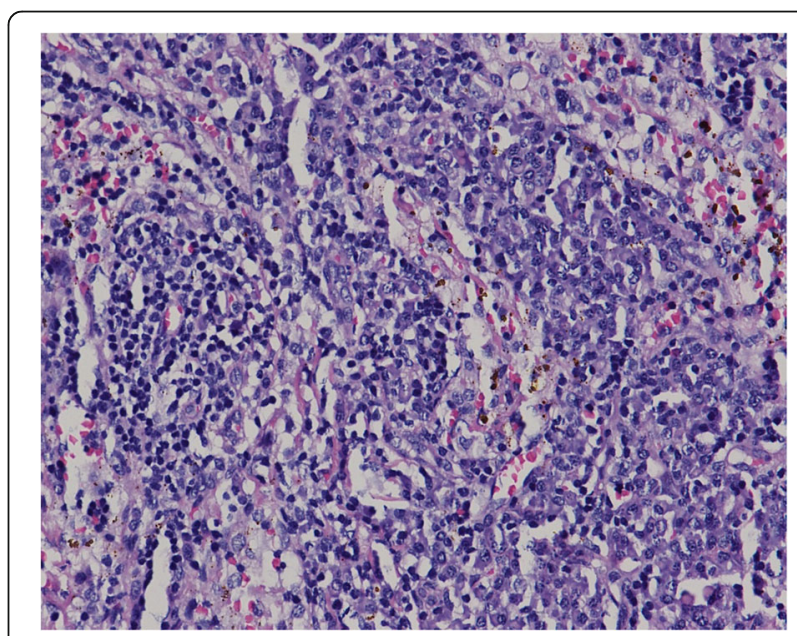

Fig. 2 Histopathology of liver biopsy (Hematoxylin-Eosin staining, 100x). The histopathology revealed swelling in part of the hepatocyte, spotty necrosis occasionally and a small quantity of lymphocyte infiltration in portal area, with no evidence of cirrhosis 


\section{Discussion}

Portal hypertension is rare in POEMS syndrome. Hepatomegaly is the most common hepatic manifestation in POEMS syndrome [4] and $39.6 \% \sim 54 \%$ of patients have ascites [5, 6]. In most cases, characteristics of ascites is non-portal hypertensive and the average SAAG is $6.7 \mathrm{~g} / \mathrm{l}$ [5]. While in this case, SAAG of ascites fluid is over $11 \mathrm{~g} / \mathrm{l}$, accompanied by splenomegaly and esophageal and gastric varices. All evidence confirmed the presence of portal hypertension.

In this case, portal hypertension was probably associated with POEMS syndrome. Since there are no investigations suggested the presence of cirrhosis or other common causes of portal hypertension. Moreover, portal hypertension improved greatly after the systemic treatment of POEMS syndrome. Though the mechanism underlying POEMS syndrome and portal hypertension remains unknown, the association may be not occasional.

The mechanism of portal hypertension occurring in POEMS syndrome is unknown. It was reported that various pathogenic determinants including progressive fibrosis of the portal veins, acquired vascular defect, exposure to several toxins, infections, immunological basis, aberrations in the thrombin-antithrombin complex were related to portal hypertension [7]. Many inflammatory cytokines such as VEGF, Interleukin-1 $\beta$, Interleukin-6, Interleukin-12 [8], tumor necrosis factor- $\alpha$ [9] are increased in POEMS syndrome. VEGF induces endothelial cells proliferation, migration and microvascular hyperpermeability,and play an important role in angiogenesis [10]. In this case, the VEGF level was significantly elevated in the early stages. Perhaps microvascular hyperpermeability following VEGF overproduction contributes to the onset of portal hypertension. A hepatic circulation defect secondary to POEMS syndrome may contribute to the subsequent portal hypertension.

Melphalan combined with dexamethasone has comparable response rates with autologous peripheral blood stem cell transplantation (PBSCT) [11]. Novel agents such as lenalidomide [12] and bortezomib [13] are suitable for relapsed patients and patients with severe organ dysfunction. But there is no random control trials and multicenter international cooperation.

\section{Conclusion}

This case highlights the manifestation of portal hypertension in POEMS syndrome. Lenalidomide with or without dexamethasone is effective for portal hypertension due to POEMS syndrome, though esophageal and gastric varices seem not reversible so easily.

\section{Abbreviations}

PBSCT: Peripheral blood stem cell transplantation; SAAG: Serum-ascites albumin gradient; VEGF: Vascular endothelial growth factor.

\section{Acknowledgements}

We kindly acknowledge Yuxia Guan for excellent nursing care in the course of treatment.

Funding

Not applicable.

Availability of data and materials

ALL of the data were collected by chart and can be available from the corresponding author upon reasonable request.

\section{Authors' contributions}

LW collected the clinical data and drafted the article. YL initiated the paper and revised the manuscript critically for important intellectual content. YF, CL and JQ analyzed the date in the manuscript and mentored the study. JL and $Y L$ conducted the crucial treatment protocols, and therapy was followed the guidance of $J \mathrm{~L}$ and $\mathrm{YL}$. WZ analyzed the histopathology of cervical lymph nodes and liver of the patient, which contributed to the final diagnosis. All of the authors report no conflicts of interest and approved of the final version.

\section{Competing interests}

The authors declare that they have no competing interests.

\section{Consent for publication}

Written informed consent was obtained from the patient for publication of this case report and any accompanying images. A copy of the written consent is available for review by the Editor of this journal.

Ethics approval and consent to participate

The study was approved by the Ethics Committee of Peking Union Medical College Hospital.

\section{Publisher's Note}

Springer Nature remains neutral with regard to jurisdictional claims in published maps and institutional affiliations.

\section{Author details}

${ }^{1}$ Department of Gastroenterology, Peking Union Medical College Hospital, Chinese Academy of Medical Sciences and Peking Union Medical College, Beijing 100730, China. ${ }^{2}$ Department of Hematology, Peking Union Medical College Hospital, Chinese Academy of Medical Sciences and Peking Union Medical College, Beijing 100730, China. ${ }^{3}$ Department of Pathology, Peking Union Medical College Hospital, Chinese Academy of Medical Sciences and Peking Union Medical College, Beijing 100730, China.

Received: 26 October 2015 Accepted: 2 April 2017

Published online: 11 May 2017

\section{References}

1. Bardwick PA, Zvaifler NJ, Gill GN, Newman D, Greenway GD, Resnick DL. Plasma cell dyscrasia with polyneuropathy, organomegaly, endocrinopathy, $M$ protein, and skin changes: the POEMS syndrome. Report on two cases and a review of the literature. Medicine. 1980;59:311-22.

2. Inoue R, Nakazawa A, Tsukada N, Katoh Y, Nagao T, Nakanuma Y, et al. POEMS syndrome with idiopathic portal hypertension: autopsy case and review of the literature. Pathol Int. 2010;60:316-20.

3. Campos S, Agostinho C, Cipriano MA. POEMS syndrome and idiopathic portal hypertension: a possible association. Revista espanola de enfermedades digestivas : organo oficial de la Sociedad Espanola de Patologia Digestiva. 2017. doi:10.17235/reed.2017.4623/2016.

4. Dispenzieri A. POEMS syndrome: 2014 update on diagnosis, riskstratification, and management. Am J Hematol. 2014;89:214-23.

5. Cui RT, Yu SY, Huang XS, Zhang JT, Li F, Pu CQ. The characteristics of ascites in patients with POEMS syndrome. Ann Hematol. 2013;92:1661-4.

6. Li J, Zhou DB, Huang Z, Jiao L, Duan MH, Zhang W, et al. Clinical characteristics and long-term outcome of patients with POEMS syndrome in China. Ann Hematol. 2011;90:819-26.

7. Kondo F. Benign nodular hepatocellular lesions caused by abnormal hepatic circulation: etiological analysis and introduction of a new concept. J Gastroenterol Hepatol. 2001;16:1319-28. 
8. Kanai K, Sawai S, Sogawa K, Mori M, Misawa S, Shibuya K, et al. Markedly upregulated serum interleukin-12 as a novel biomarker in POEMS syndrome. Neurology. 2012;79:575-82.

9. Rose C, Zandecki M, Copin MC, Gosset P, Labalette M, Hatron PY, et al. POEMS syndrome: report on six patients with unusual clinical signs, elevated levels of cytokines, macrophage involvement and chromosomal aberrations of bone marrow plasma cells. Leukemia. 1997;11:1318-23.

10. Watanabe O, Arimura K, Kitajima I, Osame M, Maruyama I. Greatly raised vascular endothelial growth factor (VEGF) in POEMS syndrome. Lancet. 1996;347:702

11. Li J, Zhang W, Jiao L, Duan MH, Guan HZ, Zhu WG, et al. Combination of melphalan and dexamethasone for patients with newly diagnosed POEMS syndrome. Blood. 2011;117:6445-9.

12. Tomas JF, Giraldo P, Lecumberri R, Nistal S. POEMS syndrome with severe neurological damage clinically recovered with lenalidomide. Haematologica. 2012;97:320-2

13. Li J, Zhang W, Kang WY, Cao XX, Duan MH, Zhou DB. Bortezomib and dexamethasone as first-line therapy for a patient with newly diagnosed polyneuropathy, organomegaly, endocrinopathy, M protein and skin changes syndrome complicated by renal failure. Leuk Lymphoma. 2012;53:2527-9.

Submit your next manuscript to BioMed Central and we will help you at every step:

- We accept pre-submission inquiries

- Our selector tool helps you to find the most relevant journal

- We provide round the clock customer support

- Convenient online submission

- Thorough peer review

- Inclusion in PubMed and all major indexing services

- Maximum visibility for your research

Submit your manuscript at www.biomedcentral.com/submit
Biomed Central 